\title{
Fluorescence navigation with indocyanine green for identification of intersegmental planes using a photodynamic eye camera
}

\author{
Kazuhito Funai, Akikazu Kawase, Kei Shimizu, Keigo Sekihara, Takashi Yamashita, Norihiko Shiiya \\ First Department of Surgery, Hamamatsu University School of Medicine, Hamamatsu, Japan \\ Contributions: (I) Conception and design: All authors; (II) Administrative support: K Funai, N Shiiya; (III) Provision of study materials or patients: \\ K Funai, A Kawase, K Shimizu, K Sekihara, T Yamashita; (IV) Collection and assembly of data: K Funai, A Kawase; (V) Data analysis and \\ interpretation: K Funai, A Kawase; (VI) Manuscript writing: All authors; (VII) Final approval of manuscript: All authors. \\ Correspondence to: Kazuhito Funai, MD, PhD. First Department of Surgery, Hamamatsu University School of Medicine, 1-20-1, Handayama, \\ Higashi-ku, Hamamatsu, Shizuoka 431-3192, Japan. Email: kfunai@hama-med.ac.jp.
}

\begin{abstract}
Background: Pulmonary segmentectomy is an important surgical option for complete resection in patients with poor lung function. However, correctly recognizing the intersegmental plane for accurate segmentectomy is sometimes difficult. We therefore developed a novel method that allows the detection of intersegmental planes using an indocyanine green (ICG) fluorescence imaging device, photodynamic eye
\end{abstract} (PDE) camera, PDE-neo.

Methods: As a prospective study, we performed bronchial ICG-guided segmentectomy using PDE-neo. The patients were placed in a lateral position under general anesthesia, and we performed a combined muscle-sparing minithoracotomy with video assistance. The pulmonary artery, pulmonary vein, and segmental bronchi were separated, and ICG mixed with autologous blood was introduced by spraying through the resected segment bronchi to enable visualization of the intersegmental surface with PDEneo. This study protocol was approved by the Research Ethics Board of Hamamatsu University School of Medicine, Japan. Written informed consent was obtained from all patients.

Results: Overall, 10 lung malignancy patients, including 8 males and 2 females, participated in this study from March 2011 to October 2013. The median age was 69 years (range, 29-76 years). Pathologic diagnoses were 7 adenocarcinomas, 1 adenosquamous carcinoma, 1 carcinoid tumor, and 1 lung metastasis from the parotid gland cancer. The intersegmental planes of 8 cases could be identified by this method using a PDEneo, whereas those of 2 cases did not show clear demarcations. The reason was that because of severe emphysema, air flowed from the resected segment to the surrounding segments, obliterating the demarcation between the two segmental planes. There were no recurrent cases and only two deaths due to other diseases were observed; and the 5-year cause-specific survival rate was $100 \%$.

Conclusions: Intersegmental planes could be more easily identified using ICG fluorescence imaging during segmentectomy. This method is feasible and effective and has a good long-term prognosis.

Keywords: Emphysema; fluorescence imaging; indocyanine green (ICG); lung neoplasms; segmentectomy

Submitted Mar 23, 2020. Accepted for publication Jul 25, 2020.

doi: $10.21037 /$ jtd-20-1448

View this article at: http://dx.doi.org/10.21037/jtd-20-1448

\section{Introduction}

Since the demonstration of the superiority of lobectomy over sublobar lung resection for lung cancer in 1995 by the Lung Cancer Study Group (1), pulmonary segmentectomy has been recognized as an operative option for complete resection in patients with poor pulmonary function $(2,3)$, although lobectomy and pneumonectomy remain the standard operations for patients with primary lung cancer. Elderly patients who are heavy smokers are far more likely to develop lung cancer and emphysema; therefore, 


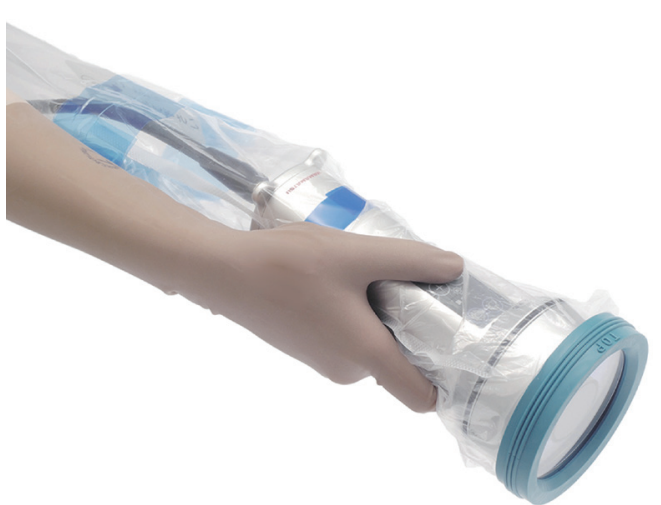

Figure 1 PDE-neo consists of a camera unit, a controller, and a remote controller; the camera unit measures $80 \times 182 \times 80 \mathrm{~mm}$ (width, depth, height). In the surgical field, it is used in a dedicated sterilized drape. (Extracted from the catalog).

opportunities to perform segmentectomy in them are increasing. The key to pulmonary segmentectomy is to recognize the intersegmental plane correctly. One common method for this identification is the inflation/ deflation technique $(4,5)$ wherein a demarcation line is intraoperatively identified by inflation/deflation of the target segment by clamping and unclamping the relevant bronchus. However, although the inflation/deflation technique is effective in identifying the demarcation line on the pleural surface, it is ineffective for deep parts of the lung.

Indocyanine green (ICG) fluorescence imaging is a technique that uses a medical charge-coupled device (CCD) camera to capture the near-infrared light emitted when ICG binds to $\alpha 1$ lipoprotein in the blood, thereby enabling the observation of tissues in a living body. When ICG is injected into the body and excited by infrared light $(760 \mathrm{~nm})$, infrared fluorescence $(830 \mathrm{~nm})$ is generated, which is then captured using a photodynamic eye (PDE) and observed using a contrast (6). ICG fluorescence imaging has been used in various fields in recent years because of its minimal side effects, minimal invasiveness, and simplicity, and its usefulness has been reported. To the best of our knowledge, the first use of ICG in pulmonary segmentectomy was the experimental animal study reported by Misaki et al. in 2009 (7). Subsequently, they reported the technology of visualizing the demarcation line using an infrared thoracoscopic system in eight human cases in 2010 (8). They administered ICG intravenously; however, segmentectomy is usually performed based on bronchial anatomy, and accordingly transbronchial introduction of ICG is ideal. Therefore, we have begun a prospective study examining the safety and utility of bronchial ICG-guided video-assisted thoracoscopic segmentectomy.

In this study, we present a novel method that allows the detection of intersegmental planes using ICG fluorescence imaging.

We present the following article in accordance with the MDAR reporting checklist (available at http://dx.doi. org/10.21037/jtd-20-1448).

\section{Methods}

This study is a joint research by Hamamatsu University School of Medicine and Hamamatsu Photonics. Fluorescence imaging was performed using PDE-neo (Hamamatsu Photonics, Hamamatsu, Japan) that was loaned from Hamamatsu Photonics, as defined by the joint research agreement. The study protocol was approved by the Research Ethics Board of Hamamatsu University School of Medicine, Japan [22-117]. Written informed consent was obtained from all patients. The study was conducted in accordance with the Declaration of Helsinki (as revised in 2013). The University Medical Information Network (UMIN) clinical trials registry number is UMIN000006679.

The near-infrared fluorescence imaging system, PDEneo, is equipped with a light emitting diode that emits near-infrared wavelength of $760 \mathrm{~nm}$ as excited light and a CCD camera as an image detector with an optical highpass filter in front of it so that fluorescence signals can be efficiently detected (6). PDE-neo consists of a camera unit, a controller, and a remote controller; the camera unit measures $80 \times 182 \times 80 \mathrm{~mm}$ (width, depth, height). In the surgical field, it is used in a dedicated sterilized drape (Figure 1).

Bronchial ICG-guided video-assisted thoracoscopic segmentectomy was performed using PDE-neo in 10 patients with lung malignancy. Nine patients had primary lung cancer and one had metastatic lung tumor. The 9 patients with primary lung cancer underwent segmental resection via passive reduction surgery owing to poor lung function and complications. We made a surgical plan to have a margin equal to or larger than the tumor diameter.

The cases were pathologically staged based on the $8^{\text {th }}$ edition of the TNM classification for lung cancer (9). Histopathological studies were done according to the criteria outlined by the World Health Organization (10). Overall survival was estimated using the Kaplan-Meier 


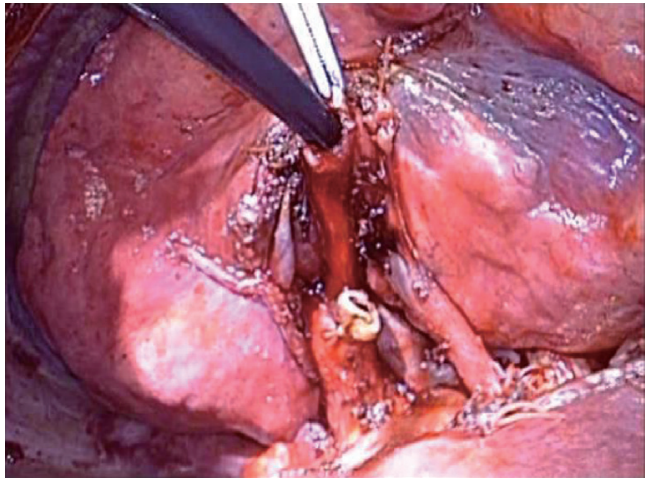

Figure 2 A mixture of ICG and autologous blood was introduced into the resected segmental bronchial stump $(\mathrm{S} 2+3)$ by mixing them with air using a spray set for fibrin glue (BOLHEAL ${ }^{\circledR}$ Spray Set; Akita Sumitomo Bakelite Co., Ltd., Akita, Japan) (Case 2).

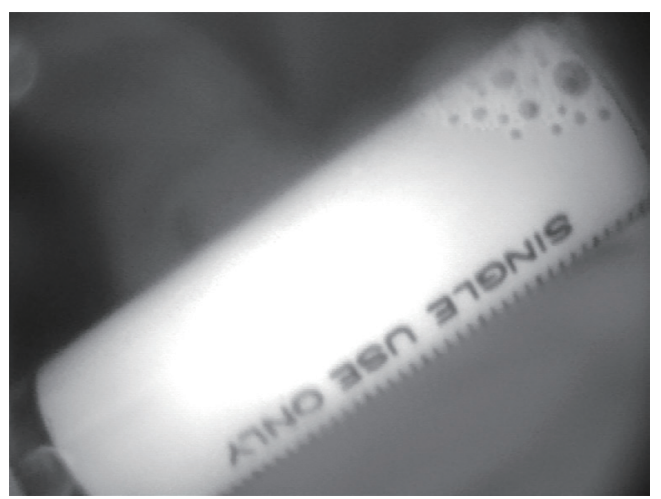

Figure 3 A volume of $5 \mathrm{~mL}$ each of ICG and autologous blood were mixed. The mixture emits fluorescence when observed with PDE.

method, and zero time was set as the date of pulmonary resection. The endpoint of overall survival was defined as the date of death from any cause, and cause-specific survival was defined as the date of cancer-specific death. The last follow-up observation was censored when the patient was alive. All statistical analyses were performed using the SPSS statistics software package version 25 (IBM SPSS statistics).

\section{Operative technique}

After administering general anesthesia, a Bronco-Cath ${ }^{\mathrm{TM}}$ endobronchial tube (Medtronic, USA) was introduced. Thereafter, the patient was placed in a lateral position and we performed operation with combined muscle-sparing minithoracotomy and video assistance. The appropriate pulmonary veins and arteries were separated using staplers

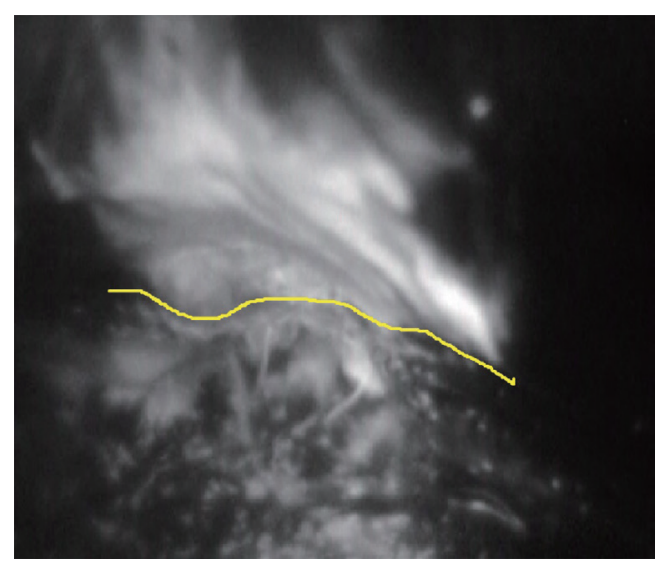

Figure 4 Determination of the intersegmental demarcation line by ICG fluorescence, PDE-neo, is shown. The segment to be resected is displayed in white.

or by direct ligation. After stapling and disconnecting the segmental bronchus, a mixture of ICG and autologous blood was introduced into the resected segmental bronchial stump by mixing them with air using a spray set for fibrin glue (BOLHEAL ${ }^{\circledR}$ Spray Set; Akita Sumitomo Bakelite Co., Ltd. Akita, Japan) (Figure 2). $5 \mathrm{~mL}$ each of ICG and autologous blood were mixed and used. Sekine et al. (11) used 5 -fold saline-diluted ICG, but we used $5 \mathrm{~mL}$ each of ICG and autologous blood that was more visible in PDE (Figure 3). The segment to be resected was gradually inflated, and it glowed white when observed using PDEneo (Figure 4). Thereafter, the intersegmental planes were separated using staples or electrical cautery under the guidance of PDE-neo.

\section{Results}

In total, 10 lung malignancy patients, including 8 males and 2 females, participated in this study from March 2011 to October 2013. The median age of the patients was 69 (range, 29-76) years. Nine patients had primary lung cancer and one had metastatic lung tumor. Pathologic diagnoses were 7 adenocarcinomas, 1 adenosquamous carcinoma, 1 carcinoid tumor, and 1 lung metastasis from the parotid gland cancer (Table 1). The use of ICG had no effect on pathological diagnosis. All of them had clinical indications for pulmonary segmentectomy. The nine patients with primary lung cancer underwent segmental resection via passive limited surgery because of poor lung function and complications. 
Table 1 Patient characteristics

\begin{tabular}{|c|c|c|c|c|c|c|c|c|c|c|}
\hline Case & $\begin{array}{c}\text { Age } \\
\text { (years) }\end{array}$ & Sex & Diagnosis & $\begin{array}{l}\text { Smoking } \\
\text { index }\end{array}$ & FEV1.0\% & Comorbidity & TNM & $\begin{array}{c}\text { Tumor } \\
\text { diameter }\end{array}$ & $\begin{array}{l}\text { Solid or } \\
\text { not solid }\end{array}$ & $\begin{array}{l}\text { Location of } \\
\text { the tumor }\end{array}$ \\
\hline 1 & 75 & $M$ & $\mathrm{Ad}-\mathrm{Sq}$ & 1,200 & 76 & $\mathrm{DM}, \mathrm{CKD}$ & T1aNoM0 & 22 & Solid & S10 \\
\hline 3 & 73 & M & Ad & 430 & 68 & & T1aNOMO & 17 & Solid & $\mathrm{S} 1+2$ \\
\hline 6 & 60 & $\mathrm{~F}$ & Carcinoid & 0 & 76 & HT, Cushing synd. & T1aNOMO & 10 & Solid & S6 \\
\hline 7 & 29 & $M$ & Metastasis & 160 & 85 & $\begin{array}{c}\text { Parotid adenocarcinoma, } \\
\text { Arrhythmia }\end{array}$ & - & 5 & Solid & S3 \\
\hline 8 & 76 & $\mathrm{~F}$ & Ad & 0 & 82 & Breast cancer, HT & T1cNOMO & 9 & Solid & S6 \\
\hline 10 & 70 & $\mathrm{M}$ & Ad & 0 & 98 & MM & T1aNoMo & 19 & Part-solid & s9 \\
\hline
\end{tabular}

DM, diabetes mellitus; CKD, chronic kidney disease; GGN, ground-glass nodule; ICH, intracerebral hemorrhage; ML, malignant lymphoma; $\mathrm{HT}$, hypertension; MM, malignant melanoma.

Segmentectomy regions included 1 right $\mathrm{S} 2+\mathrm{S} 3$ segment (anteroposterior segment of the upper lobe), 3 left upper division segments, 1 left lingual segment, 1 right S6 segment (the superior segment of the lower lobe), 2 left S6 segments, and 2 left basal segment. Separation between the segmental planes was performed using a stapler in 1 patient, electrical cautery in 2 patients, and a combination of both in 7 patients. The pathological stage of the primary lung cancer was IA1 (T1aN0M0) in 6 cases, 1A2 (T1bN0M0) in 2 , and $1 \mathrm{~A} 3$ (T1cNOM0) in one. The median operation time was 203 minutes (range, 151-254 minutes), and the median blood loss was $25 \mathrm{~mL}$ (micro-240 mL). The postoperative duration of drainage had a median of 3.5 days (1-11 days), and the postoperative hospital stay was 10.5 days (7-65 days) (Table 1).

There were no complications resulting from ICG transbronchial administration. Our method is effective for deep parts of the lung. The intersegmental planes of 8 cases could be identified using PDE and those of 2 cases could not be identified. This was due to severe emphysema because the air flowed from the resected segment to the surrounding segment, making identification of the two segmental planes impossible. Therefore, in these 2 cases, we used the traditional segmentectomy technique; an incision was made between the anatomical segmental planes separated by the pulmonary veins (12).

There was a mild air leak from the area surface separated by the electric knife. However, control was possible using soft coagulation, PGA sheets, and fibrin glue (Table 2). However, other complications were observed in 5 cases: two cases of pneumonia (Grade I and II), two wound infections (Grade I and IIIb), and one prolonged air leak (Grade IIIa); However, serious complications of ClavienDindo classification (13) III or higher were found in 2 cases (Table 2). Pneumonia occurred in the different lobe from the remaining pulmonary segment. The operation time was longer in complicated segmentectomy cases.

Long-term prognosis after pulmonary segmentectomy was good. The median follow-up period was 80 months (range, 1-91 months). There were no recurrences, and only two deaths due to other diseases were observed. These were secondary to postoperative acute exacerbation of interstitial pneumonia after discharge and respiratory failure associated with exacerbation of aspergillosis 50 months after surgery. The 5-year cause-specific survival rate was 100\% (Figure 5), and the 5 -year overall survival rate was $79 \%$ (Figure 6).

\section{Discussion}

Compared with lobectomy, limited resection has three-times the local recurrence rate and a poor prognosis (1), therefore, it is especially important to secure the surgical margin in segmentectomy. For that reason, the most important part of segmentectomy is the correct identification of the 


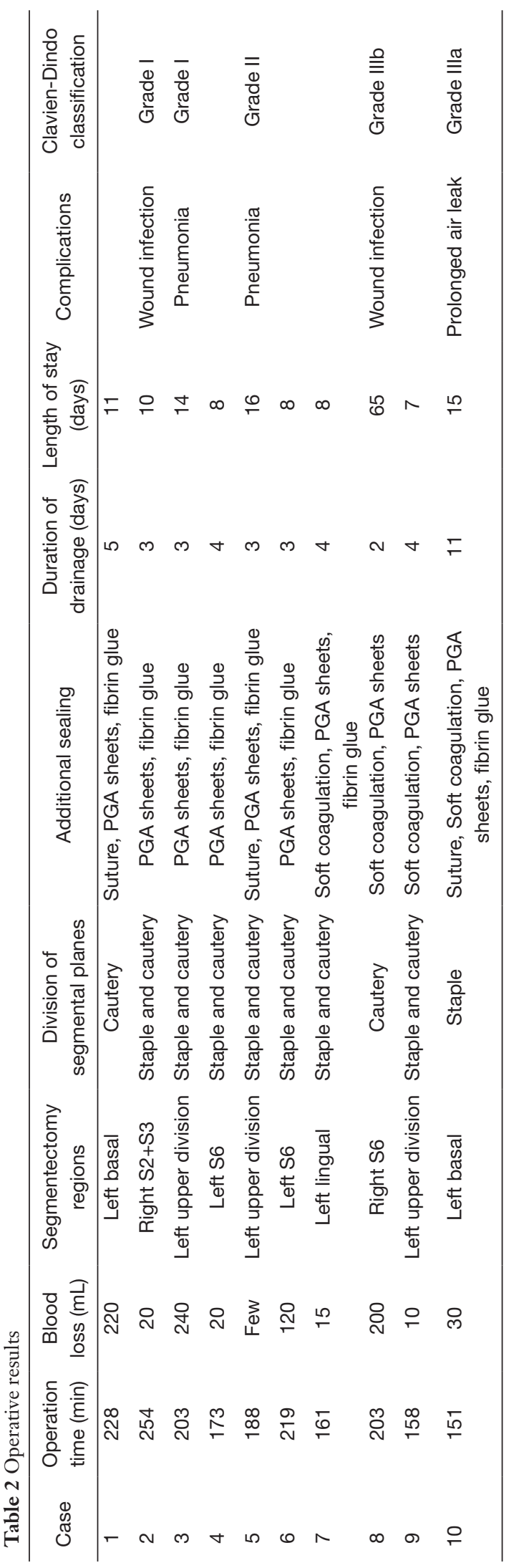

intersegmental planes between the segment for resection and the healthy segments. Multiple methods have been proposed to identify these intersegmental planes during pulmonary segmentectomy. There are advantages and disadvantages with each method, and rapid technological progress has resulted in changes in strategies. One common method for lung structure segmentation is the air inflation technique $(4,5)$ where the intersegmental plane is identified based on the difference in air content between the segment to be excised and the segment to be left. However, one of the major limitations to this method is that the border can be obscured or misleading because of collateral ventilation, which results in inflation of the area that is supposed to be left deflated. This problem depends on the condition of the lung, such as emphysema.

Oh et al. developed a new technique for detecting intersegmental planes using ICG instead of the conventional residual segment expansion method (14). The technique is simple and consists first of ligation of the segmental vein to prevent loss of ICG and then injection of ICG through the segmental bronchus. These two steps result in easy identification of the intersegmental planes by a change in color of the surface and of the lung parenchyma (14). However, in smokers' lungs with a high degree of anthracosis, the green color of ICG is inconspicuous, and intersegment planes may not be identified. In this study, which is a modified version of Oh's report (14), intersegmental planes were identified by spraying a mixture of ICG and autologous blood into the segment for resection through a resected segmental bronchus. This way, the intersegmental planes could be more clearly identified based on ICG fluorescence than based on ICG color difference. Identification of intersegmental planes was possible with this method in $80 \%$ of cases. Sekine et al. have reported surgical results with injecting the ICG mixture into the segmental bronchus using the PDE (11) as was used to perform the segmentectomy mentioned in this paper.

A method has been reported in which ICG is intravenously administered and the area is identified using an infrared thoracoscopic system (8). ICG fluorescence has recently been widely used in respiratory surgery, most of which are performed by intravenously administering ICG $(8,15,16)$. However, traditionally, segmentectomy has been performed based on bronchial anatomy. In addition, the lymphatic channels from the tumor are along the bronchi. Therefore, we believe that segmentectomy using transbronchial introduction of ICG is ideal. However, it is not important whether intrabronchial infusion or 


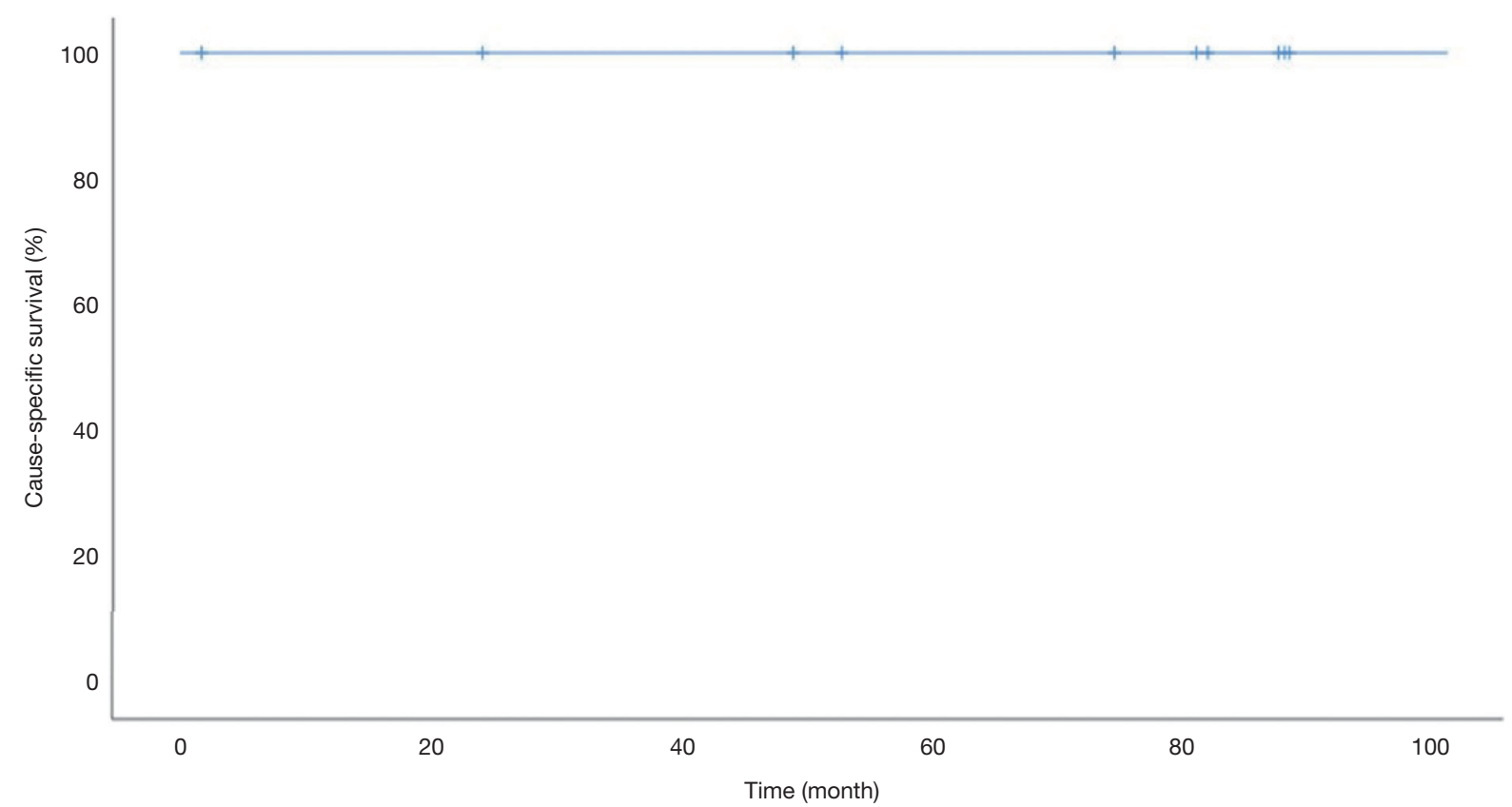

Figure 5 The 5-year cause-specific survival rate was 100\%.

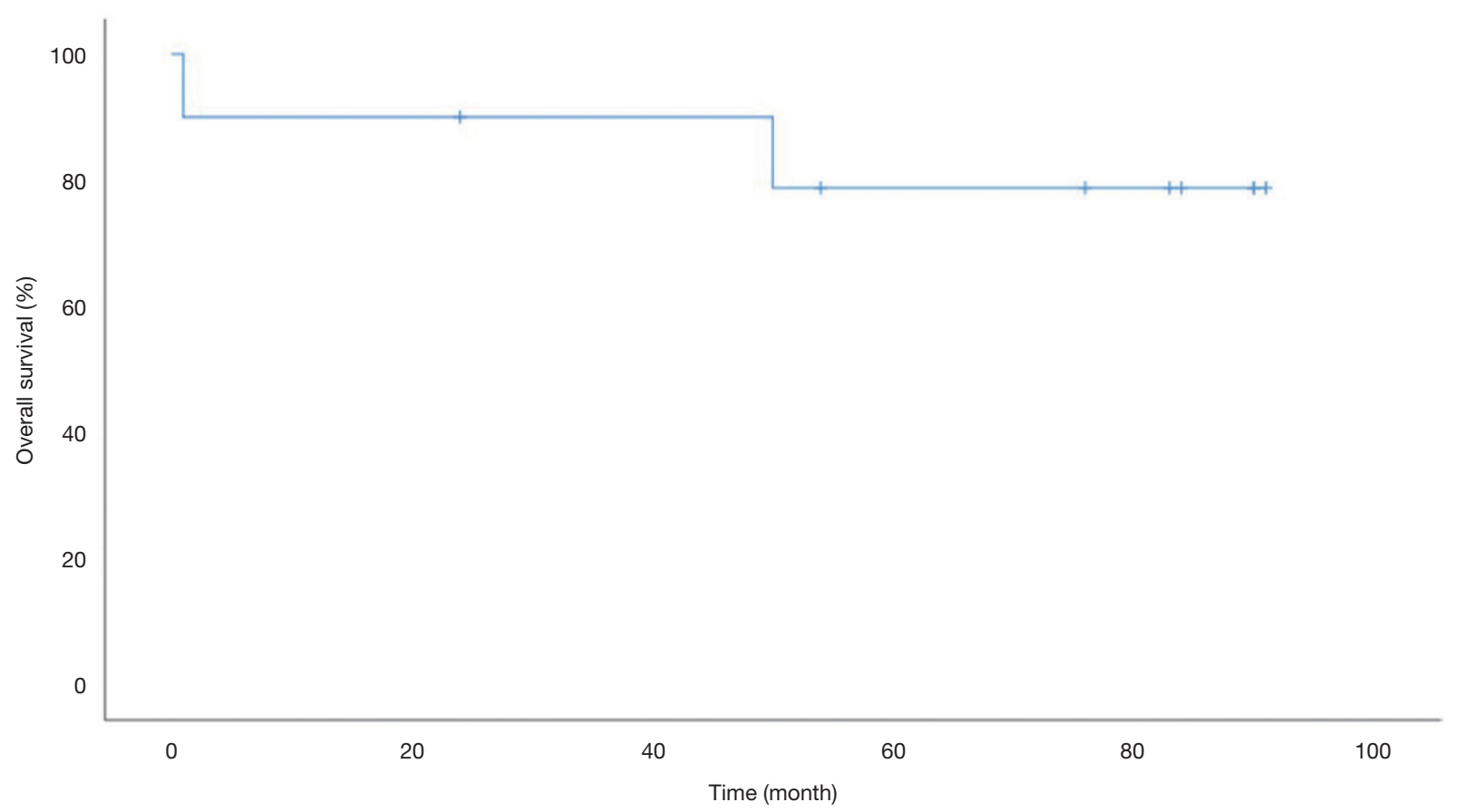

Figure 6 The 5-year overall survival rate was 79\%. Median survival time was not reached.

intravenous infusion is better; it is important to make the best use of the merits of both to identify the areas correctly to get the margin from the tumor as planned. More recently, new techniques such as thoracoscopic segmentectomy using intravenous ICG combined with ICG-Virtual-assisted lung mapping have been reported by some authors $(17,18)$. In 
addition, compared to the time when we were conducting this research, the ICG camera was also made smaller and united with the thoracoscopic camera head. It is important to select the modality that suits each case using new technologies such as multidetector CT scan with contrast enhancement and 3-Dimensional reconstructions.

Precise understanding of the segmental plane during surgery ensures the planned tumor margin results in elimination of local recurrence and improved prognosis. Previous studies have concentrated on the technical safety and efficacy but, to our best knowledge, no studies have identified long-term prognoses of using ICG fluorescence imaging for pulmonary segmentectomy. Therefore, we followed-up the cases in this prospective study for more than 5 years and reported on the results of the prognosis, which showed that PDE-neo is an excellent technique for identifying intersegmental planes and treating cancer. A 100\% 5-year cause-specific survival rate for segmental resection cases in this study indicates that segmental resection was performed successfully as planned.

A limitation of this study was that it included only a small number of patients within a single institution; therefore, it may not be enough to judge adequately the safety, feasibility, accurateness, and long-term prognosis of the procedure. Hence, for verification of our results, a larger group will be needed.

The method of using ICG fluorescence imaging is therefore a very effective technique for demarcating intersegmental planes curing lung neoplasm. However, in severe pulmonary emphysema cases, it may be difficult to identify segmental planes using this method. Therefore, based on complications, it is important to identify segmental planes reliably by choosing appropriately between our method and intravenous administration of ICG.

\section{Conclusions}

The method of using ICG fluorescence imaging during segmentectomy is therefore a very effective technique for demarcating intersegmental planes curing lung neoplasm. This method is feasible and effective and has a good longterm prognosis.

\section{Acknowledgments}

We would like to thank Editage (www.editage.com) for English language editing.

Funding: None.

\section{Footnote}

Reporting Checklist: The authors have completed the MDAR reporting checklist. Available at http://dx.doi.org/10.21037/ jtd-20-1448

Data Sharing Statement: Available at http://dx.doi. org/10.21037/jtd-20-1448

Peer Review File: Available at http://dx.doi.org/10.21037/jtd20-1448

Conflicts of Interest: All authors have completed the ICMJE uniform disclosure form (available at http://dx.doi. org/10.21037/jtd-20-1448). The authors have no conflicts of interest to declare.

Ethical Statement: The authors are accountable for all aspects of the work in ensuring that questions related to the accuracy or integrity of any part of the work are appropriately investigated and resolved. The study protocol was approved by the Research Ethics Board of Hamamatsu University School of Medicine, Japan [22-117]. Written informed consent was obtained from all patients. The study was conducted in accordance with the Declaration of Helsinki (as revised in 2013). The University Medical Information Network (UMIN) clinical trials registry number is UMIN000006679.

Open Access Statement: This is an Open Access article distributed in accordance with the Creative Commons Attribution-NonCommercial-NoDerivs 4.0 International License (CC BY-NC-ND 4.0), which permits the noncommercial replication and distribution of the article with the strict proviso that no changes or edits are made and the original work is properly cited (including links to both the formal publication through the relevant DOI and the license). See: https://creativecommons.org/licenses/by-nc-nd/4.0/.

\section{References}

1. Ginsberg RJ, Rubinstein LV. Randomized trial of lobectomy versus limited resection for T1 N0 non-small cell lung cancer. Lung Cancer Study Group. Ann Thorac Surg 1995;60:615-22; discussion 622-3.

2. Pastorino $\mathrm{U}$, Valente $\mathrm{M}$, Bedini $\mathrm{V}$, et al. Limited resection for Stage I lung cancer. Eur J Surg Oncol 1991;17:42-6.

3. Martin-Ucar AE, Nakas A, Pilling JE, et al. A case- 
matched study of anatomical segmentectomy versus lobectomy for stage I lung cancer in high-risk patients. Eur J Cardiothorac Surg 2005;27:675-9.

4. Overholt RH, Woods FM, Betts RH. An improved method of resection of pulmonary segments; report of a technique applied in 70 operations. J Thorac Surg 1948;17:464-79.

5. Rubenstein LH, O'Neill TJ, Glover RP. A technique for pulmonary segmental delineation. J Thorac Surg 1949;18:75-81.

6. Shikayama T. Characteristics of the photodynamic eye camera. In: Kusano M, Kokudo N, Toi M, et al. editors. ICG fluorescence imaging and navigation surgery. Tokyo: Springer, 2016:21-7.

7. Misaki N, Chang SS, Gotoh M, et al. A novel method for determining adjacent lung segments with infrared thoracoscopy. J Thorac Cardiovasc Surg 2009;138:613-8.

8. Misaki N, Chang SS, Igai H, et al. New clinically applicable method for visualizing adjacent lung segments using an infrared thoracoscopy system. J Thorac Cardiovasc Surg 2010;140:752-6.

9. Goldstraw P, Chansky K, Crowley J, et al. The IASLC Lung Cancer Staging Project: Proposals for revision of the TNM stage groupings in the forthcoming (eighth) edition of the TNM classification for lung cancer. J Thorac Oncol 2016;11:39-51.

10. Travis WD, Brambilla E, Burke A, et al. WHO classification of tumours of the lung, pleura, thymus and heart. International Agency for Research on Cancer; 2015.

11. Sekine Y, Ko E, Oishi H, et al. A simple and effective technique for identification of intersegmental planes

Cite this article as: Funai K, Kawase A, Shimizu K, Sekihara K, Yamashita T, Shiiya N. Fluorescence navigation with indocyanine green for identification of intersegmental planes using a photodynamic eye camera. J Thorac Dis 2020;12(9):48174824. doi: $10.21037 /$ jtd-20-1448 by infrared thoracoscopy after transbronchial injection of indocyanine green. J Thorac Cardiovasc Surg 2012;143:1330-5.

12. LoCicero J. Segmentectomy and lesser pulmonary resections. In: Shields TW, LoCicero J, Ponn RB. General thoracic surgery. Philadelphia, PA; London: Lippincott Williams \& Wilkins, 2000.

13. Dindo D, Demartines N, Clavien PA. Classification of surgical complications: a new proposal with evaluation in a cohort of 6336 patients and results of a survey. Ann Surg 2004;240:205-13.

14. Oh S, Suzuki K, Miyasaka Y, et al. New technique for lung segmentectomy using indocyanine green injection. Ann Thorac Surg 2013;95:2188-90.

15. Oh Y, Quan YH, Kim M, et al. Intraoperative fluorescence image-guided pulmonary segmentectomy. J Surg Res 2015;199:287-93.

16. Iizuka S, Kuroda H, Yoshimura K, et al. Predictors of indocyanine green visualization during fluorescence imaging for segmental plane formation in thoracoscopic anatomical segmentectomy. J Thorac Dis 2016;8:985-91.

17. Sato M, Omasa M, Chen F, et al. Use of virtual assisted lung mapping (VAL-MAP), a bronchoscopic multispot dye-marking technique using virtual images, for precise navigation of thoracoscopic sublobar lung resection. J Thorac Cardiovasc Surg 2014;147:1813-9.

18. Yang SM, Lin CK, Chen LW, et al. Combined virtualassisted lung mapping (VAL-MAP) with CT-guided localization in thoracoscopic pulmonary segmentectomy. Asian J Surg 2019;42:488-94. 